\title{
Successful Treatment of Super-Refractory Status Epilepticus With Cannabis Oil in a Patient With Neuronal Ceroid Lipofuscinosis
}

\author{
Athena Michaelides ${ }^{\mathrm{a}, \mathrm{b}}$, Spyridon Bekas ${ }^{\mathrm{a}}$, Antigoni Papaioannou ${ }^{\mathrm{a}}$, \\ Athina Lazaridou ${ }^{\mathrm{a}}$, Paola Nicolaides ${ }^{\mathrm{a}}$
}

\begin{abstract}
Herein we describe a case of a 25-year-old man diagnosed with the "variant late infantile-onset" neuronal ceroid lipofuscinosis (NCL) who has a novel mutation in the CLN6 gene located on chromosome 15. The patient developed seizures at the age of 10 years along with progressive symptoms of ataxia, spasticity, cognitive decline and visual difficulties. At the age of 25 years, the seizures were drug-resistant, and the patient was put in an anesthesia-induced coma until the use of cannabis oil in combination with Food and Drug Administration (FDA)-approved anti-seizure drugs proved to be lifesaving. Treatment for NCL currently consists of symptomatic relief of seizures, and supportive therapies for associated symptoms of behavior, language and visual difficulties. Although limited, recent data on the efficacy of cannabinoids for treatment-resistant epilepsy have been published. Since cannabis oil is not currently FDA approved for most formulations, its use in drug-resistant epilepsy is anecdotal and remains highly controversial.
\end{abstract}

Keywords: Epilepsy; Drug-resistant seizure; Neuronal ceroid lipofuscinosis; CLN6

\section{Introduction}

Neuronal ceroid lipofuscinosis (NCL) is a group of genetically inherited neurodegenerative disorders. There are 14 genetically distinct types that have been identified and are classified as CLN1 to CLN14. Most NCLs have an autosomal recessive pattern of inheritance and share clinical characteristics. The childhood types are more common and manifest as progressive vision loss, epileptic seizures, mental and motor deterioration, and premature death [1].

Manuscript submitted August 11, 2020, accepted November 19, 2020

Published online December 9, 2020

aUniversity of Nicosia Medical School, Engomi 2408, Cyprus

${ }^{\mathrm{b}}$ Corresponding Author: Athena Michaelides, University of Nicosia Medical

School, Engomi 2408, Cyprus. Email: athena1.michaelides@gmail.com

doi: https://doi.org/10.14740/jnr626
The management of NCL is challenging, and often requires different treatment regimens for the range of presenting symptoms. A prominent feature reported across the literature is epilepsy [2-5]. Since different anti-seizure drugs are not always beneficial in reducing seizures, an alternative treatment that has recently been recognized to be efficacious is cannabis oil in differing ratios of cannabidiol (CBD) and tetrahydrocannabinol (THC) [6, 7]. Apart from Epidiolex (purified form of nearly $100 \%$ CBD), cannabis oil is not currently Food and Drug Administration (FDA) approved, so its use is controversial [6].

\section{Case Report}

We describe a case of a 25-year-old man, who presented with drug-resistant epilepsy due to variant late infantile neuronal ceroid lipofuscinosis (vLINCL).

The patient described is the third son of healthy non-consanguineous Cypriot parents with no known family history of neurological disease. He was born following a full-term normal delivery and reached his early developmental milestone. There were no concerns until the age of 8 years when he developed mild learning difficulties thought to be related to impaired memory and concentration. A diagnosis of attention deficit hyperactivity disorder (ADHD) was made, and methylphenidate (ritalin) was prescribed.

At the age of 10 years, he developed his first focal motor seizure. A few months later, he had a second brief right-sided focal motor seizure with an electroencephalogram (EEG) pattern suggestive of benign childhood epilepsy with centrotemporal spikes (BCECTS), also known as benign rolandic epilepsy. At that point, ritalin was withdrawn and sodium valproate (depakine) was added.

Over the course of the year, he developed a progressive loss of motor skills and coordination - he experienced frequent falls, increasing difficulty with walking and climbing stairs, tremor and ataxia. His speech became slower and dysarthric. A brain magnetic resonance imaging (MRI) showed moderate dilation of the ventricles and extra-axial cerebrospinal fluid (CSF) spaces with minimal periventricular hyper intensities. The corpus collosum, brain stem and cervical spinal cord appeared normal. Genetic testing for spinocerebellar ataxia (SCA) excluded a diagnosis of SCA types 1, 2, 3, 6, 7, 8, 12 and 17, as well as dentatorubral-pallidoluysian atrophy (DRPLA). 
His symptoms were progressive still and at the age of 14 years he was found to have proximal muscle weakness in the upper and lower limbs, and increased tone in the lower limbs with brisk reflexes and ankle clonus. He had a spastic gait ataxia with early development of scoliosis and some asymmetry at the pelvis. He also had obvious dysarthria with frequent choking episodes. On ophthalmic examination, he had failure of binocular convergence and nystagmus that was elicited on lateral gaze. Repeat MRI showed diffuse hypomyelination in the cerebrum in conjunction with cerebellar atrophy. The thalamus and globus pallidi were not thought to be atrophic.

His seizures continued to progress and remained drug-resistant requiring the use of a number of anti-seizure medications including a combination of levetiracetam, lamotrigine, lacosamide and piracetam. At the age of 22 years, genetic testing confirmed two novel variations in the CLN6 gene: a c. $407 \mathrm{G}>\mathrm{A}$ in exon 4 and a c. $884 \mathrm{~A}>\mathrm{G}$ in exon 7 resulting in the p. Arg136His and p. Tyr295Cys amino acid changes at the protein level.

The above variations were not listed in either the CLN6 gene mutation database or single nucleotide polymorphism (SNP) databases. Upon genetic testing of both parents and brothers, only the mother was found to be a carrier of the CLN6 gene $\mathrm{c} 407 \mathrm{G}>$ A mutation with a $50 \%$ chance of transmitting the mutation to her offspring. The c. $407 \mathrm{G}>\mathrm{A}$ mutation was thus inherited from the mother and the c. $884 \mathrm{~A}>\mathrm{G}$ variation appeared de novo in the proband.

No curative treatments exist for the NCLs, so despite a formal diagnosis of vLINCL, no definitive treatment could be administered. The seizures progressed to super-refractory status epilepticus (SRSE) resistant to phenobarbital, clobazam, sodium valproate, phenytoin, levetiracetam and zonisamide. The patient was admitted to the intensive care unit (ICU) and put in an anesthesia-induced coma using midazolam and propofol and remained so for a period of several weeks. Any attempt to reduce or reverse the anesthesia-induced coma resulted in the re-emergence of status epilepticus. At this point, a trial of cannabis oil was initiated with improvement in the clinical and electrographic seizures. Over the following 2 weeks, he was trialled on a combined form of CBD and THC. A significant reduction in epileptic seizures was observed within a few days of adding the $\mathrm{CBD} / \mathrm{THC}$ combination. Following a further increase and after 50 days of being in the induced coma, the sedation medication was withdrawn completely, and the patient opened his eyes following a week without epileptic episodes. The patient was discharged home 4 weeks following a reduction of the anti-seizure drugs and daily intake of $\mathrm{CBD} / \mathrm{THC}$.

Currently 8 months on, the patient experiences a significantly reduced number of seizures. He has come off clobazam and zonizamide but continues taking the cannabinoid oil drop combination in addition to low dose phenobarbitone, sodium valproate and levetiracetam.

\section{Discussion}

The NCLs are caused by a number of CLN gene mutations. These genes encode proteins that are involved in the secretory and/or endo/lysosomal pathways [8,9]. When these genes are mutated, auto-fluorescent lipopigments accumulate in various tissues contributing to the signs and symptoms seen in NCL [9].

CLN6 encodes an endoplasmic reticulum (ER) membrane protein [9]. The CLN6 mutation described in the case occurs on chromosome 15q21-23 on gene CLN6 [8].

\section{Spectrum and symptoms}

Different types of NCLs are described based on the ultrastructure of membrane-bound inclusions, genetic molecular defect and clinical phenotype [10]. Compared to the classical forms, the age of onset in vLINCL tends to be later with symptoms presenting as late as 6 years of age. Seizures, ataxia, irritability and progressive mental deterioration present early on. Loss of speech occurs at around 3 years, visual impairment at 4 years and loss of motor skills between 4 and 10 years [10]. The rate of disease progression may vary, but usually leads to severe disability by mid-adolescence and death by the end of the second decade in life $[4,5,9]$.

The CLN6 variant of vLINCL was first identified in 2002 in Costa Rican and Venezuelen patients [9]. Since then, other cases have been reported that describe a disease course and progression similar to our case. Sun et al reported the first case of a CLN6 mutation in China - a male patient who initially presented with language articulation problems and uncoordinated movements from age 4 which went unrecognized until the patient had repeated seizure episodes [2]. Sato et al reported a male patient from Japan diagnosed with moderate mental retardation and hyperactivity disorder by the age of 6 years. Epilepsy followed, along with weakness, ataxia, and MRI findings of atrophy. Genetic testing at the age of 12 years revealed a heterozygous mutation in the CLN6 gene [3]. Al-Muhaizea et al reported four cases across three families in Saudi Arabia where a similar disease course was reported: signs of language and behavior regression between the ages of 2 and 4 years followed by gait deterioration, ataxia, seizures and brain atrophy at around 6 years of age [4]. Chin et al reported similar findings due to novel CLN6 mutations found in two patients of Caucasian and mixed native American/Northern European descent, respectively [5]. The cases describe a consistent pattern of disease progression; however, a differentiating feature amongst the reported cases is the presence of visual impairment - although it is described amongst the typical features of VLINCL, it only presents as a feature of the disease in certain cases [4].

\section{Treatment}

Treatment for NCL due to a CLN6 mutation currently consists of symptomatic relief of seizures, and supportive therapies for associated symptoms of behavior, language and visual difficulties.

Although limited, recent data on the efficacy of cannabinoids for treatment-resistant epilepsy have been published. Hausman-Kedem et al prospectively investigated the use of 
$\mathrm{CBD} / \mathrm{THC}$ (ratio 20:1) in children and adolescents aged 1 - 20 years with drug-resistant epilepsy to at least four anti-seizure drugs, a ketogenic diet and vagal nerve stimulation. A significant reduction in mean monthly seizure frequency was noted with a higher CBD dose and patient age $<10$ years at treatment-onset being associated with a better outcome. The most significant adverse event recorded was somnolence which led to cessation in some cases [6]. Porcari et al conducted a retrospective study that analyzed seizure frequency after the addition of CBD to anti-seizure drugs in treatment-resistant epilepsy in a cohort aged $<18$ years. Results showed a significant reduction in overall seizure frequency. Although no patients had monotherapy with $\mathrm{CBD}$, weaning of other anti-seizure drugs was reported as an achievable outcome [7].

The mechanism of cannabis in treating epilepsy remains unclear, and except for Epidiolex, FDA approval for most formulations has not yet been achieved. This forces acquisition of the drug through marijuana dispensaries which creates discrepancies in dosing and $\mathrm{CBD} / \mathrm{THC}$ ratios [6].

\section{Conclusion}

Recent developments for the management of drug-resistant epilepsy report on the use of cannabis oil in combination with anti-seizure drugs. Since cannabis oil is not currently FDA approved other than for specific epilepsy syndromes (Dravet and Lennox Gastuax), its use in drug-resistant epilepsy is anecdotal and remains highly controversial. Although this case has shown that it is beneficial in reducing the seizures in NCL, future studies are required to test the efficacy and safety of cannabis oil as well as to define a recommended ratio of $\mathrm{CBD} /$ $\mathrm{THC}$ to be trialed in drug-resistant status epilepticus.

\section{Acknowledgments}

None to declare.

\section{Financial Disclosure}

The University of Nicosia provided funding to support the publication of this manuscript.

\section{Conflict of Interest}

None to declare.

\section{Informed Consent}

Informed consent was obtained from the patient and the patient's family for the undertaking of this study. Informed consent was obtained from all family members who underwent investigational gene testing during the work up of the patient's case. Informed consent was obtained from each family member at the time of testing. In addition, the patient and the patient's family have consented to the publication of this manuscript.

\section{Author Contributions}

AM, SB, AL and AP were responsible for the data collection, writing, analysis and submission of the manuscript; PN contributed to supervising and reviewing at all stages.

\section{Data Availability}

The authors declare that data supporting the findings of this study are available within the article.

\section{References}

1. Haltia M, Goebel HH. The neuronal ceroid-lipofuscinoses: a historical introduction. Biochim Biophys Acta. 2013;1832(11):1795-1800.

2. Sun G, Yao F, Tian Z, Ma T, Yang Z. A first CLN6 variant case of late infantile neuronal ceroid lipofuscinosis caused by a homozygous mutation in a boy from China: a case report. BMC Med Genet. 2018;19(1):177.

3. Sato R, Inui T, Endo W, Okubo Y, Takezawa Y, Anzai M, Morita H, et al. First Japanese variant of late infantile neuronal ceroid lipofuscinosis caused by novel CLN6 mutations. Brain Dev. 2016;38(9):852-856.

4. Al-Muhaizea MA, Al-Hassnan ZN, Chedrawi A. Variant late infantile neuronal ceroid lipofuscinosis (CLN6 gene) in Saudi Arabia. Pediatr Neurol. 2009;41(1):74-76.

5. Chin JJ, Behnam B, Davids M, Sharma P, Zein WM, Wang C, Chepa-Lotrea X, et al. Novel mutations in CLN6 cause late-infantile neuronal ceroid lipofuscinosis without visual impairment in two unrelated patients. Mol Genet Metab. 2019;126(2):188-195.

6. Hausman-Kedem M, Menascu S, Kramer U. Efficacy of CBD-enriched medical cannabis for treatment of refractory epilepsy in children and adolescents - An observational, longitudinal study. Brain Dev. 2018;40(7):544-551.

7. Porcari GS, Fu C, Doll ED, Carter EG, Carson RP. Efficacy of artisanal preparations of cannabidiol for the treatment of epilepsy: Practical experiences in a tertiary medical center. Epilepsy Behav. 2018;80:240-246.

8. Mole SE, Cotman SL. Genetics of the neuronal ceroid lipofuscinoses (Batten disease). Biochim Biophys Acta. 2015;1852(10 Pt B):2237-2241.

9. Wheeler RB, Sharp JD, Schultz RA, Joslin JM, Williams $\mathrm{RE}$, Mole SE. The gene mutated in variant late-infantile neuronal ceroid lipofuscinosis (CLN6) and in nclf mutant mice encodes a novel predicted transmembrane protein. Am J Hum Genet. 2002;70(2):537-542.

10. Teixeira CA, Espinola J, Huo L, Kohlschutter J, Persaud Sawin DA, Minassian B, Bessa CJ, et al. Novel mutations in the CLN6 gene causing a variant late infantile neuronal ceroid lipofuscinosis. Hum Mutat. 2003;21(5):502-508. 